\title{
THE
}

2008

\section{Statistically Interacting Quasiparticles in Ising Chains}

\author{
Ping Lu \\ University of Rhode Island \\ Jared Vanasse \\ University of Rhode Island \\ Christopher Piecuch \\ University of Rhode Island \\ Michael Karbach \\ University of Rhode Island \\ Gerhard Müller \\ University of Rhode Island, gmuller@uri.edu
}

Follow this and additional works at: https://digitalcommons.uri.edu/phys_facpubs

Terms of Use

All rights reserved under copyright.

\section{Citation/Publisher Attribution}

Ping Lu et al 2008 J. Phys. A: Math. Theor. 41265003

Available at: http://dx.doi.org/10.1088/1751-8113/41/26/265003

This Article is brought to you for free and open access by the Physics at DigitalCommons@URI. It has been accepted for inclusion in Physics Faculty Publications by an authorized administrator of DigitalCommons@URI. For more information, please contact digitalcommons-group@uri.edu. 


\title{
Statistically interacting quasiparticles in Ising chains
}

\author{
Ping Lu ${ }^{1}$, Jared Vanasse ${ }^{1}$, Christopher Piecuch ${ }^{1}$, Michael \\ Karbach $^{1,2}$, and Gerhard Müller ${ }^{1}$ \\ ${ }^{1}$ Department of Physics, University of Rhode Island, Kingston RI 02881, USA \\ ${ }^{2}$ Fachbereich Physik, Bergische Universität Wuppertal, 42097 Wuppertal, \\ Germany \\ PACS numbers: $75.10 .-b$
}

\begin{abstract}
The exclusion statistics of two complementary sets of quasiparticles, generated from opposite ends of the spectrum, are identified for Ising chains with spin $s=1 / 2,1$. In the $s=1 / 2$ case the two sets are antiferromagnetic domain walls (solitons) and ferromagnetic domains (strings). In the $s=1$ case they are soliton pairs and nested strings, respectively. The Ising model is equivalent to a system of two species of solitons for $s=1 / 2$ and to a system of six species of soliton pairs for $s=1$. Solitons exist on single bonds but soliton pairs may be spread across many bonds. The thermodynamics of a system of domains spanning up to $M$ lattice sites is amenable to exact analysis and shown to become equivalent, in the limit $M \rightarrow \infty$, to the thermodynamics of the $s=1 / 2$ Ising chain. A relation is presented between the solitons in the Ising limit and the spinons in the $X X$ limit of the $s=1 / 2 X X Z$ chain.
\end{abstract}

\section{Introduction}

Ising chains are among the simplest systems of interacting degrees of freedom and have been thoroughly studied in a wide variety of circumstances including the presence of transverse fields, time-dependent fields, inhomogeneities in field or coupling etc. Is there anything of substance left that we can still learn from the Ising model in one dimension [1] with homogeneous coupling? This paper presents a case (by no means the only one [2, 3]) for an affirmative answer.

The Hamiltonian of the spin- $s$ Ising model for $s=1 / 2,1,3 / 2, \ldots$ on a periodic chain of $N$ sites reads

$$
\mathcal{H}_{s}=\sum_{n=1}^{N}\left(J S_{n}^{z} S_{n+1}^{z}+h S_{n}^{z}\right), \quad S_{n}^{z}=s, s-1, \ldots,-s,
$$

where the exchange coupling is antiferromagnetic (ferromagnetic) for $J>0(J<0)$ and $h$ is a magnetic field. This model system has simple product eigenstates, a dispersionless spectrum, and no intrinsic dynamics to speak of. Its thermodynamics, derived via transfer matrix [4, 5], is predictably simple.

One interesting aspect of $\mathcal{H}_{s}$ that promises usefulness in a wider context is the quasiparticle composition of the product eigenstates as will be demonstrated. The entire spectrum of $\mathcal{H}_{s}$ can be systematically generated from opposite ends by different sets of quasiparticles with exotic exclusion statistics. The nature of these quasiparticles strongly varies with $s$ but a systematics in their make-up is recognizable. 
In $\mathcal{H}_{1 / 2}$ we consider antiferromagnetic domain walls (solitons with spin $\pm 1 / 2$ and fractional exclusion statistics) for $J>0$ or with ferromagnetic domains (strings of flipped spins with integer-valued exclusion statistics) for $J<0$. The corresponding quasiparticles in $\mathcal{H}_{1}$ turn out to be soliton pairs with spin $0, \pm 1$ (for $J>0$ ) and nested strings (for $J<0$ ), both with unusual exclusion statistics.

We use the concept of statistically interacting quasiparticles to show that the thermodynamics of $\mathcal{H}_{s}$ is equivalent to that of a gas of solitons (for $s=1 / 2$ ) or soliton pairs (for $s=1$ ). The same framework is shown to work also for the thermodynamics of string particles. It is expected that these particles, whose detailed exclusion statistics is worked out here, are still relevant in integrable spin chain models away from their Ising limit. The particles identified here then become objects of a coordinate Bethe ansatz [6, 7, 8, applied to those models.

We first review the concept of statistical interaction and its use in thermodynamics (Sec. 21). Then we introduce the soliton particles for $\mathcal{H}_{1 / 2}$, describe their exclusion statistics, and determine the thermodynamics in a magnetic field from a soliton perspective (Sec. 3). Next we introduce the six species of soliton-pair particles that govern the spectrum of $\mathcal{H}_{1}$ and work out their thermodynamics in zero magnetic field (Sec. 4). Then we present the combinatorics for the statistical interaction of a system of strings in $\mathcal{H}_{1 / 2}$ and of nested strings in $\mathcal{H}_{1}$. We proceed by calculating the thermodynamics of a system of strings of restricted size and recover the Ising thermodynamics when that restriction is lifted (Sec. 55). Finally, we assess the progress reported here and discuss possible extensions and comparisons (Sec. 6) including a relation between solitons and spinons, both with semionic statistics Appendix A .

\section{Statistical interaction}

Quasiparticles in solid matter are not restricted to be either bosons or fermions. In integrable quantum many-body model systems [6, 7, 8, quasiparticles with infinite lifetimes and unusual exclusion statistics have indeed been identified. The generalized Pauli principle as introduced by Haldane [9] expresses how the number of states available to one particle is affected by the presence of other particles:

$$
\Delta d_{i} \doteq-\sum_{j} g_{i j} \Delta N_{j}
$$

The indices $i, j$ refer to distinct particle species. The $g_{i j}$ are statistical interaction coefficients. For bosons we have $g_{i j}=0$ and for fermions $g_{i j}=\delta_{i j}$. Upon integration Eq. (2.1) becomes

$$
d_{i}=A_{i}-\sum_{j} g_{i j}\left(N_{j}-\delta_{i j}\right)
$$

where the $A_{i}$ are statistical capacity constants. The number of many-body states containing $\left\{N_{i}\right\}$ particles of the various species is then determined by the multiplicity expression

$$
W\left(\left\{N_{i}\right\}\right)=\prod_{i}\left(\begin{array}{c}
d_{i}+N_{i}-1 \\
N_{i}
\end{array}\right) .
$$

We shall determine the ingredients $A_{i}, g_{i j}$ to (2.2) for two species of solitons or $N$ species of strings, all pertaining to $\mathcal{H}_{1 / 2}$, and for six species of soliton pairs or $N(N+1) / 2$ species of nested strings pertaining to $\mathcal{H}_{1}$. 
The thermodynamic properties of a macroscopic system of statistically interacting particles are amenable to a rigorous analysis as shown by $\mathrm{Wu}[10$. For given sets of 1-particle energies $\epsilon_{i}$, statistical interaction coefficients $g_{i j}$, and statistical capacity constants $A_{i}$, the grand partition function is

$$
Z=\prod_{i}\left[\frac{1+w_{i}}{w_{i}}\right]^{A_{i}}
$$

where the quantities $w_{i}$ are determined by the nonlinear algebraic equations

$$
\frac{\epsilon_{i}-\mu}{k_{B} T}=\ln \left(1+w_{i}\right)-\sum_{j} g_{j i} \ln \left(\frac{1+w_{j}}{w_{j}}\right) \text {. }
$$

The temperature $T$ and the chemical potential $\mu$ are the control variables. Additional control variables such as external fields may come into play as part of the energies $\epsilon_{i}$. The average numbers of particles, $\left\langle N_{i}\right\rangle$, of each species are related to the $w_{i}$ by the linear equations

$$
w_{i}\left\langle N_{i}\right\rangle+\sum_{j} g_{i j}\left\langle N_{j}\right\rangle=A_{i}
$$

We shall apply this method of exact analysis to the solitons and the strings in the context of $\mathcal{H}_{1 / 2}$ and to soliton pairs in the context of $\mathcal{H}_{1}$.

\section{Solitons}

Here we consider $\mathcal{H}_{1 / 2}$ with $J>0$ and $h>0$ for even or odd $N$. The task at hand has a combinatorial part and a statistical mechanical part. We first relate the Ising spectrum to soliton configurations, then we undertake a thermodynamic analysis of the soliton system, using the methodology outlined in Sec. 2.

\subsection{Combinatorics of solitons}

Among the four distinct bonds in the general product state $\left|\sigma_{1} \sigma_{2} \cdots \sigma_{N}\right\rangle$ (see Table 1), the bonds $\uparrow \uparrow, \downarrow \downarrow$ represent solitons with spin $+1 / 2,-1 / 2$, respectively, and $\uparrow \downarrow, \downarrow \uparrow$ are vacuum bonds. Close-packed solitons with like spin orientation reside on successive bonds (e.g. $\uparrow \uparrow \uparrow)$, whereas close-packed solitons with opposite spin orientation are separated by one vacuum bond (e.g. $\uparrow \uparrow \downarrow \downarrow$ ). More generally, the number of vacuum bonds between nearest-neighbor solitons with like (opposite) spin orientation is even (odd). Solitons only interact statistically. The energy of a soliton is unaffected by the presence of other solitons.

Table 1. Distinct bonds in $\mathcal{H}_{1 / 2}$, their soliton content, and their contribution to the energy of the product eigenstate (relative to the soliton vacuum).

\begin{tabular}{c|cccc} 
bond & $\uparrow \uparrow$ & $\downarrow \downarrow$ & $\uparrow \downarrow$ & $\downarrow \uparrow$ \\
\hline$N_{+}$ & 1 & 0 & 0 & 0 \\
$N_{-}$ & 0 & 1 & 0 & 0 \\
\hline$\Delta E$ & $\frac{J+h}{2}$ & $\frac{J-h}{2}$ & 0 & 0
\end{tabular}


The two soliton vacuum states, $|\uparrow \downarrow \cdots \uparrow \downarrow\rangle$ and $|\downarrow \uparrow \cdots \downarrow \uparrow\rangle$, represent the lowest energy level for even $N$. The lowest level for odd $N$ is $2 N$-fold degenerate and contains one soliton. The soliton content of an Ising eigenstate is specified either by the numbers $N_{ \pm}$of spin-up/down solitons or, alternatively, by the total number of solitons and the magnetisation:

$$
N_{A}=N_{+}+N_{-}, \quad M_{z}=\frac{1}{2}\left(N_{+}-N_{-}\right) .
$$

The energy level of all states with $N_{A}$ solitons and magnetisation $M_{z}$ is

$$
E\left(N_{A}, M_{z}\right)-E_{0}=\frac{1}{2} N_{A} J+h M_{z},
$$

where $E_{0}=-N J / 4$ is the energy of the soliton vacuum.

How many Ising eigenstates exist for given $N_{+}$and $N_{-}$(or $N_{A}$ and $M_{z}$ )? The solution of this combinatorial problem is the following multiplicity expression constructed from extensive tabulated data such as sampled in Table 2 .

$$
\begin{aligned}
& W_{A}\left(N_{+}, N_{-}\right)=\frac{2 N}{N-N_{A}} \prod_{\sigma= \pm}\left(\begin{array}{c}
d_{\sigma}+N_{\sigma}-1 \\
N_{\sigma}
\end{array}\right), \\
& d_{\sigma}=\frac{1}{2}(N-1)-\frac{1}{2} \sum_{\sigma^{\prime}}\left(N_{\sigma^{\prime}}-\delta_{\sigma \sigma^{\prime}}\right) .
\end{aligned}
$$

It is indeed compatible with the standard form (2.3). The range of $N_{A}$ is $0,2, \cdots, N$ for even $N$ and $1,3, \cdots, N$ for odd $N . N_{A}=N$ is only realized for the two states with $N_{+}=N_{A}$ or $N_{-}=N_{A}$. This multiplicity expression specifies the statistical interaction between soliton particles.

Table 2. Number of states, $W_{A}\left(N_{+}, N_{-}\right)$, with $N_{A}=N_{+}+N_{-}$solitons and magnetisation $M_{z}=\left(N_{+}-N_{-}\right) / 2$ for $\mathcal{H}_{1 / 2}$ with $N=6$ (left) and $N=7$ (right).

\begin{tabular}{r|cccc|c}
$M_{z} \backslash N_{A}$ & 0 & 2 & 4 & 6 & \\
\hline 3 & - & - & - & 1 & 1 \\
2 & - & - & 6 & - & 6 \\
1 & - & 9 & 6 & - & 15 \\
0 & 2 & 12 & 6 & - & 20 \\
-1 & - & 9 & 6 & - & 15 \\
-2 & - & - & 6 & - & 6 \\
-3 & - & - & - & 1 & 1 \\
\hline & 2 & 30 & 30 & 2 & 64
\end{tabular}

\begin{tabular}{c|cccc|c}
$M_{z} \backslash N_{A}$ & 1 & 3 & 5 & 7 & \\
\hline $7 / 2$ & - & - & - & 1 & 1 \\
$5 / 2$ & - & - & 7 & - & 7 \\
$3 / 2$ & - & 14 & 7 & - & 21 \\
$1 / 2$ & 7 & 21 & 7 & - & 35 \\
$-1 / 2$ & 7 & 21 & 7 & - & 35 \\
$-3 / 2$ & - & 14 & 7 & - & 21 \\
$-5 / 2$ & - & - & 7 & - & 7 \\
$-7 / 2$ & - & - & - & 1 & 1 \\
\hline & 14 & 70 & 42 & 2 & 128
\end{tabular}

\subsection{Statistical mechanics of solitons}

For the statistical mechanical analysis of $\mathcal{H}_{1 / 2}$ as a soliton gas we use the statistical capacity constants $A_{\sigma}=(N-1) / 2$ and the statistical interaction coefficients $g_{\sigma \sigma^{\prime}}=$ $1 / 2$ extracted from Eq. (3.4), and the soliton energies $\epsilon_{\sigma}=(J+\sigma h) / 2$ from Table 1 We have to solve two coupled nonlinear algebraic equations of the type (2.5):

$$
\frac{J \pm h}{2 k_{B} T}=\ln \left(1+w_{ \pm}\right)+\frac{1}{2} \ln \frac{w_{ \pm}}{1+w_{ \pm}}+\frac{1}{2} \ln \frac{w_{\mp}}{1+w_{\mp}} .
$$


The solution,

$$
w_{ \pm}=\frac{1}{2}\left[e^{ \pm h / k_{B} T}-1+\sqrt{\left(e^{ \pm h / k_{B} T}-1\right)^{2}+4 e^{(J \pm h) / k_{B} T}}\right],
$$

determines the grand partition function via (2.4) with the asymptotic value $A_{ \pm} \rightsquigarrow N / 2$ for the capacity constants. The result,

$$
Z=\left[e^{2 K}\left(\cosh H+\sqrt{\sinh ^{2} H+e^{-4 K}}\right)\right]^{N}, \quad K \doteq-\frac{J}{4 k_{B} T}, \quad H \doteq-\frac{h}{2 k_{B} T},
$$

is in exact agreement with the well-known canonical partition function $Z_{N}$ obtained via transfer-matrix [4]. In the relation $Z=Z_{N} e^{N K}$, the factor $e^{N K}$ accounts for the relative shift of energy scales used in the two methods.

For the average numbers of solitons, $\left\langle N_{ \pm}\right\rangle$, we infer from (2.6) the two coupled linear equations,

$$
\left(w_{ \pm}+\frac{1}{2}\right)\left\langle N_{ \pm}\right\rangle+\frac{1}{2}\left\langle N_{\mp}\right\rangle=\frac{N}{2},
$$

which have the solutions

$$
\left\langle N_{ \pm}\right\rangle=\frac{N}{2} \frac{e^{ \pm H}\left[\sqrt{\sinh ^{2} H+e^{-4 K}} \pm \sinh H\right]}{\sinh ^{2} H+e^{-4 K}+\cosh H \sqrt{\sinh ^{2} H+e^{-4 K}}} \stackrel{h \rightarrow 0}{\longrightarrow} \frac{N / 2}{e^{-2 K}+1} .
$$

The dependence of $\left\langle N_{+}\right\rangle / N$ on $J / k_{B} T$ is shown in Fig. 1 1 for various values of $h / J$. All curves start from $\left\langle N_{+}\right\rangle / N=1 / 4$ in the high- $T$ limit. For $h=0$ we have $\left\langle N_{+}\right\rangle=\left\langle N_{-}\right\rangle$; this curve has a monotonically decreasing trend toward zero as $T \rightarrow 0$. For $h>0$ $(h<0)$ the average number $\left\langle N_{+}\right\rangle$of solitons with spin directed antiparallel (parallel) to $h$ is more (less) rapidly suppressed as $T \rightarrow 0$. For sufficiently weak, negative fields, $0>h / J>-0.25$, the curve is still monotonically decreasing. For $-0.25>h / J>-1$, it acquires a smooth maximum at finite, nonzero $T$. For $h / J<-1$ the curve is monotonically increasing toward $\left\langle N_{+}\right\rangle / N=1$. Here the ground state contains $N$ spinpolarized solitons.

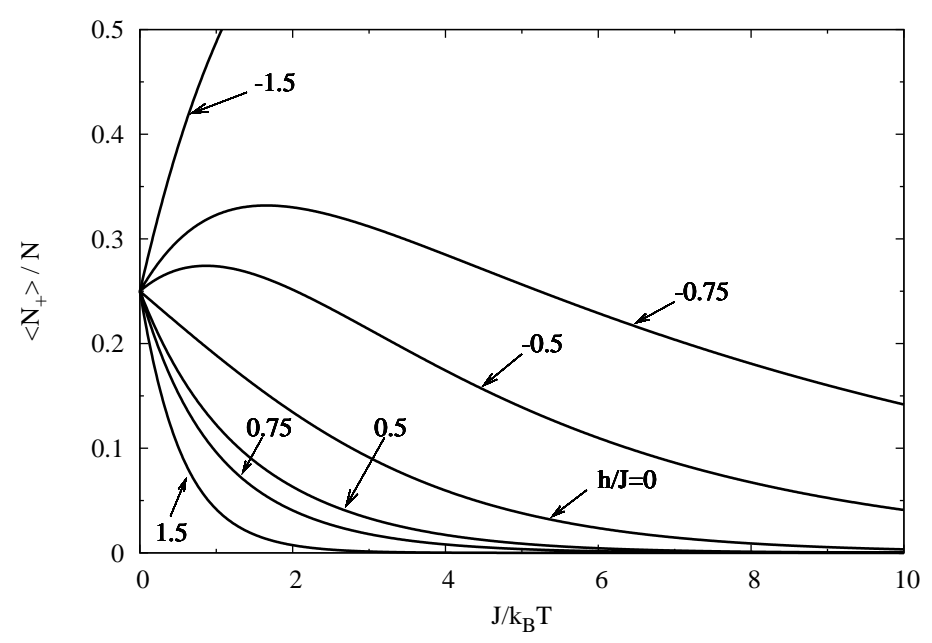

Figure 1. Average number $\left\langle N_{+}\right\rangle / N$ of spin-up solitons per lattice bond versus $J / k_{B} T$ for several values of magnetic field. 


\section{Soliton pairs}

Here we consider $\mathcal{H}_{1}$ with $J>0$ and $h=0$ for even or odd $N$. The generalization to $h \neq 0$ is straightforward conceptually. The independent particles are now soliton pairs.

\subsection{Combinatorics of soliton pairs}

The nine different kinds of bonds are listed in Table 3. Each bond can accommodate up to two solitons. The energy of a soliton is not the same in all configurations.

Table 3. Distinct bonds in $\mathcal{H}_{1}$, their soliton content, and their contribution to the energy of a product eigenstate (relative to the soliton vacuum).

\begin{tabular}{c|ccccccccc} 
bond & $\uparrow \uparrow$ & $\circ \circ$ & $\downarrow \downarrow$ & $\uparrow \circ$ & $\circ \uparrow$ & $\downarrow \circ$ & $\circ \downarrow$ & $\uparrow \downarrow$ & $\downarrow \uparrow$ \\
\hline$N_{+}$ & 2 & 1 & 0 & 1 & 1 & 0 & 0 & 0 & 0 \\
$N_{-}$ & 0 & 1 & 2 & 0 & 0 & 1 & 1 & 0 & 0 \\
\hline$\Delta E / J$ & 2 & 1 & 2 & 1 & 1 & 1 & 1 & 0 & 0
\end{tabular}

Our search for the independent particles again starts from extensive tabulated data for $W_{A}\left(N_{+}, N_{-}\right)$such as sampled in Table 4. Several clues suggest that the independent particles are soliton pairs. For example, the number of solitons is always even. Also, the number of states with $N_{-}=0$ grows $\propto N^{N_{+} / 2}$ for $N_{+} \ll N$ as opposed to the growth $\propto N^{N_{+}}$observed in $\mathcal{H}_{1 / 2}$

The systematic examination of the data tables for $W_{A}\left(N_{+}, N_{-}\right)$points to the existence of six distinct species of soliton-pair particles, two groups of three species with spin $(+1,0,-1)$. In the first group the paired solitons are confined to the same bond. In the second group the paired solitons are deconfined. They can be on bonds with any number of lattice sites between them.

Confined-soliton pairs with spin up (named $r_{+}$) are identified by any element $\uparrow \uparrow$ in the product state. In like manner, confined-soliton pairs with spin zero (down) are

Table 4. Number of states, $W_{A}\left(N_{+}, N_{-}\right)$, with $N_{A}=N_{+}+N_{-}$solitons and magnetisation $M_{z}=\left(N_{+}-N_{-}\right) / 2$ for $\mathcal{H}_{1}$ with $N=3$ (left) and $N=4$ (right).

\begin{tabular}{r|cccc|c}
$M_{z} \backslash N_{A}$ & 0 & 2 & 4 & 6 & \\
\hline 3 & - & - & - & 1 & 1 \\
2 & - & - & 3 & - & 3 \\
1 & - & 3 & 3 & - & 6 \\
0 & - & 6 & - & 1 & 7 \\
-1 & - & 3 & 3 & - & 6 \\
-2 & - & - & 3 & - & 3 \\
-3 & - & - & - & 1 & 1 \\
\hline & 0 & 12 & 12 & 3 & 27
\end{tabular}

\begin{tabular}{r|ccccc|c}
$M_{z} \backslash N_{A}$ & 0 & 2 & 4 & 6 & 8 & \\
\hline 4 & - & - & - & - & 1 & 1 \\
3 & - & - & - & 4 & - & 4 \\
2 & - & - & 6 & 4 & - & 10 \\
1 & - & 4 & 8 & 4 & - & 16 \\
0 & 2 & - & 16 & - & 1 & 19 \\
-1 & - & 4 & 8 & 4 & - & 16 \\
-2 & - & - & 6 & 4 & - & 10 \\
-3 & - & - & - & 4 & - & 4 \\
-4 & - & - & - & - & 1 & 1 \\
\hline & 2 & 8 & 48 & 20 & 3 & 81
\end{tabular}


named $r_{0}\left(r_{-}\right)$and identified by elements $\circ \circ(\downarrow \downarrow)$ in the product state. Deconfinedsoliton pairs with spin up (down) are named $q_{+}\left(q_{-}\right)$and identified by any element $\uparrow \circ \cdots \circ \uparrow(\downarrow \circ \cdots \circ \downarrow)$ in the product state, where the presence of $n=1,2, \ldots$ site variables $\circ$ between two site variables $\uparrow(\downarrow)$ indicate the presence of $n-1$ spin-zero confined-soliton pairs $\left(r_{0}\right)$. Deconfined-soliton pairs with spin zero are named $q_{0}$ and are identified by elements $\uparrow \circ \cdots \circ \downarrow$ or $\downarrow \circ \cdots \circ \uparrow$ in the product state.

A list of name, motif, and soliton content for all six species of soliton-pair particles is shown in the top three rows of Table 5. In some instances, two close-packed particles share one lattice site (e.g. $\circ \circ \circ, \uparrow \circ \uparrow \uparrow, \uparrow \circ \downarrow \circ \downarrow$ ), in other instances, there is one vacuum bond in between (e.g. $\uparrow \uparrow \downarrow \downarrow, \uparrow \circ \uparrow \downarrow \circ \uparrow$ ). The particle $r_{0}$ can only exist inside one of the particles $q_{+}, q_{0}, q_{-}$. The former is instrumental to the soliton deconfinement in the latter.

The six particles are thus naturally classified into three groups, the confinedsoliton pairs $r_{+}, r_{-}$, the deconfined-soliton pairs $q_{+}, q_{0}, q_{-}$, and the spacer particle $r_{0}$ (deconfinement agent). In a vague QCD analogy, solitons play the role of quarks, the soliton pairs $r_{+}, r_{-}, q_{+}, q_{0}, q_{-}$the role of mesons and baryons, and the spacer particle $r_{0}$ the role of gluon with opposite action.

Table 5. Specifications of particles in $\mathcal{H}_{1}$ : confined-soliton pairs $\left(r_{+}, r_{-}\right)$, spacer particle $\left(r_{0}\right)$, deconfined-soliton pairs $\left(q_{+}, q_{0}, q_{-}\right)$; motif in product state; soliton content; index $m$ used in 4.1); statistical capacity constants $A_{m}$; energies $\epsilon_{m}$.

\begin{tabular}{r||cc|c|ccc} 
particle & $r_{+}$ & $r_{-}$ & $r_{0}$ & $q_{+}$ & $q_{-}$ & $q_{0}$ \\
\hline motif & $\uparrow \uparrow$ & $\downarrow \downarrow$ & $\circ \circ$ & $\uparrow \circ \uparrow$ & $\downarrow \circ \downarrow$ & $\uparrow \circ \downarrow, \downarrow \circ \uparrow$ \\
$N_{+}+N_{-}$ & $2+0$ & $0+2$ & $1+1$ & $2+0$ & $0+2$ & $1+1$ \\
\hline$m$ & 1 & 2 & 3 & 4 & 5 & 6 \\
$A_{m}$ & $\frac{N-1}{2}$ & $\frac{N-1}{2}$ & 0 & $\frac{N}{2}-1$ & $\frac{N}{2}-1$ & $N-2$ \\
$\epsilon_{m}$ & $2 J$ & $2 J$ & $J$ & $2 J$ & $2 J$ & $2 J$
\end{tabular}

We have determined the multiplicity expression

$$
\begin{aligned}
& W_{6}\left(\left\{X_{m}\right\}\right)=\frac{2 N}{N-N_{\Sigma}} \prod_{m=1}^{6}\left(\begin{array}{c}
d_{m}+X_{m}-1 \\
X_{m}
\end{array}\right), \\
& d_{m}=A_{m}-\sum_{m^{\prime}} g_{m m^{\prime}}\left(X_{m^{\prime}}-\delta_{m m^{\prime}}\right), \\
& N_{\Sigma}=X_{1}+X_{2}+X_{3}+2\left(X_{4}+X_{5}+X_{6}\right) \leq N,
\end{aligned}
$$

for product eigenstates containing $X_{m}$ soliton pairs of species $m=1, \ldots, 6$, where the index $m$ is defined in Table 5. It confirms the independent status of the soliton-pair particles and contains the specifications of their statistical interaction. The capacity constants $A_{m}$ and the particle energies $\epsilon_{m}$ are given in Table 5 , and the interaction coefficients $g_{m m^{\prime}}$ in Table 6. Again there exist restrictions and exceptions regarding the allowed configurations $\left\{X_{m}\right\}$. We do not list them here because they have no bearing on the statistical mechanical analysis. The only model specifications needed are the quantities $A_{m}, \epsilon_{m}, g_{m m^{\prime}}$. 
Table 6. Statistical interaction coefficients $g_{m m^{\prime}}$ between soliton-pair particles as identified in Table 5.

\begin{tabular}{c|cccrrr}
$g_{m m^{\prime}}$ & 1 & 2 & 3 & 4 & 5 & 6 \\
\hline 1 & $\frac{1}{2}$ & $\frac{1}{2}$ & $\frac{1}{2}$ & 0 & 1 & $\frac{1}{2}$ \\
2 & $\frac{1}{2}$ & $\frac{1}{2}$ & $\frac{1}{2}$ & 1 & 0 & $\frac{1}{2}$ \\
3 & 0 & 0 & 0 & -1 & -1 & -1 \\
4 & $\frac{1}{2}$ & $\frac{1}{2}$ & $\frac{1}{2}$ & 1 & 1 & $\frac{1}{2}$ \\
5 & $\frac{1}{2}$ & $\frac{1}{2}$ & $\frac{1}{2}$ & 1 & 1 & $\frac{1}{2}$ \\
6 & 1 & 1 & 1 & 2 & 2 & 2
\end{tabular}

Note that the $g_{m m^{\prime}}$ include some zeros and some negative values. To make sense of these peculiarities we rewrite each of the six binomial factors of (4.1) in the form

$$
\left(\begin{array}{c}
B_{m}+\left(1-g_{m m}\right) X_{m}-Y_{m} \\
X_{m}
\end{array}\right)
$$

where

$$
B_{m} \doteq A_{m}+g_{m m}, \quad Y_{m} \doteq \sum_{m^{\prime} \neq m} g_{m m^{\prime}} X_{m^{\prime}}+1 .
$$

The maximum capacity for particles of species $m$,

$$
X_{m}^{\max }=\frac{B_{m}-Y_{m}}{g_{m m}} .
$$

is thus primarily dictated by the diagonal coefficient $g_{m m}$, but is also influenced by the off-diagonal coefficients $g_{m m^{\prime}}$ via $Y_{m}$.

If one of the off-diagonal coefficients is zero, $g_{m m^{\prime}}=0$ for $m^{\prime} \neq m$, it merely means that the presence of particles of species $m^{\prime}$ has no effect on the capacity for particles of species $m$. If one of the diagonal coefficients vanishes, $g_{m m}=0$, then (4.4) does no longer limit the capacity for particles of species $m$. This can either mean that there is no limit (as is the case for bosons) or it can mean (as is the case here for $m=3$ ) that a limit is implied by a different rule associated with (4.1).

The existence of negative off-diagonal coefficients $g_{m m^{\prime}}$ as found in Table 6 for $m=3$ and $m^{\prime}=4,5,6$ has the consequence that particles from species $m^{\prime}$ contribute negatively to $Y_{m}$. Adding particles of species $m^{\prime}$ increases the capacity of the system for particles of species $m$. This is indeed to be expected because the latter can only exist inside the former. In this instance, the Pauli exclusion principle turns into what might be called an accommodation principle.

\subsection{Statistical mechanics of soliton pairs}

Carrying out the statistical mechanical analysis of $\mathcal{H}_{1}$ as a gas of soliton pairs starts with solving six coupled nonlinear equations of the type (2.5) with $\mu=0$, the $\epsilon_{m}$ from Table 5, and the $g_{m m^{\prime}}$ from Table 6. Symmetry implies $w_{1}=w_{2}, w_{4}=w_{5}$. The remaining four equations in exponentiated form (with $K \doteq-J / k_{B} T$ ),

$$
e^{-2 K}=\frac{w_{1} w_{4} w_{6}}{\left(1+w_{4}\right)\left(1+w_{6}\right)}, \quad e^{-K}=\frac{\left(1+w_{3}\right) w_{1} w_{4} w_{6}}{\left(1+w_{1}\right)\left(1+w_{4}\right)\left(1+w_{6}\right)},
$$


$e^{-2 K}=\frac{w_{1}\left(1+w_{3}\right) w_{4}^{2} w_{6}^{2}}{\left(1+w_{1}\right) w_{3}\left(1+w_{4}\right)\left(1+w_{6}\right)^{2}}, \quad e^{-2 K}=\frac{w_{1}\left(1+w_{3}\right) w_{4} w_{6}^{2}}{\left(1+w_{1}\right) w_{3}\left(1+w_{4}\right)\left(1+w_{6}\right)}$,

can be simplified into

$$
\frac{1+w_{3}}{1+w_{1}}=e^{K}, \quad \frac{w_{3}}{w_{6}}=e^{K}, \quad w_{4}=1+w_{6}, \quad \frac{2+w_{6}}{w_{1} w_{6}}=e^{2 K},
$$

and reduced to a quadratic equation for $w_{3}$ with the (physically significant) solution

$$
w_{3}=\cosh K-\frac{1}{2}+\sqrt{\left(\cosh K-\frac{1}{2}\right)^{2}+2}
$$

The grand partition function (2.4) with the (asymptotic) $A_{m}$ from Table 5 and the solutions (4.9), (4.10) becomes

$Z=\left[\frac{\left(1+w_{1}\right)^{2}}{w_{1}^{2}} \frac{\left(1+w_{4}\right)^{2}}{w_{4}^{2}} \frac{\left(1+w_{6}\right)^{2}}{w_{6}^{2}}\right]^{N / 2}=\left[\left(1+w_{3}\right) e^{K}\right]^{N}=Z_{N} e^{K N}$,

in agreement with the transfer-matrix result for the canonical partition function $Z_{N}$ [5], where the factor $e^{K N}$ again originates from a shift in energy scale.

For the average numbers of soliton pairs, $\left\langle N_{m}\right\rangle$, we must solve six linear equations of the type (2.6) with the $w_{m}$ from (4.9), (4.10). Symmetry dictates that $\left\langle N_{1}\right\rangle=\left\langle N_{2}\right\rangle$ and $\left\langle N_{4}\right\rangle=\left\langle N_{5}\right\rangle$. The solution reads

$$
\begin{aligned}
& \left\langle N_{1}\right\rangle=\left\langle N_{2}\right\rangle=\frac{N}{2} \frac{w_{3}\left(w_{3}^{2}+2 e^{K}\right)}{\left(w_{3}+1\right)\left(w_{3}^{2} e^{-K}+4 w_{3}+2-2 e^{K}\right)}, \\
& \left\langle N_{3}\right\rangle=N \frac{2\left(w_{3}+1-e^{K}\right)}{\left(w_{3}+1\right)\left(w_{3}^{2} e^{-K}+4 w_{3}+2-2 e^{K}\right)}, \\
& \left\langle N_{4}\right\rangle=\left\langle N_{5}\right\rangle=\frac{1}{4} w_{3}\left\langle N_{3}\right\rangle, \quad\left\langle N_{6}\right\rangle=\frac{1}{2} w_{3}\left\langle N_{3}\right\rangle .
\end{aligned}
$$

The relation $\left\langle N_{6}\right\rangle=2\left\langle N_{4}\right\rangle=2\left\langle N_{5}\right\rangle$ may be anticipated on the basis of the motif shown in Table 5. The reduced averages $\left\langle N_{m}\right\rangle / N$ are plotted versus $J / k_{B} T$ in Fig. 2

Increasing the temperature from $T=0$ results in a gradual increase of average particle numbers from all species. Notice that the spacer particles $r_{0}(m=3)$, which can only exist inside particles of species $q_{+}, q_{0}, q_{-}(m=4,5,6)$ are slowest to appear in significant numbers as would be expected.

For the generalization of these results to $h \neq 0$ we must add the Zeeman contribution to the energies $\epsilon_{m}$ listed in Table 5 . The statistical mechanical analysis of soliton pairs as demonstrated here is by no means limited to the Ising Hamiltonian (1.1). We can freeze out some of the particle species by making their activation energies infinitely large, $\epsilon_{m} \rightarrow \infty$. This has the consequence that $w_{m} \rightarrow 0$ and $\left\langle N_{m}\right\rangle \rightarrow 0$. For the remaining active particles we can assign arbitrary values $\epsilon_{m}$ for their energies. For example, if we freeze out all particles except those of species $m=1,2$ then the results of Sec. 3 for $\mathcal{H}_{1 / 2}$ are, effectively, recovered.

Among the issues that must be heeded in generalizations of the calculations reported here to models with arbitrary particle energies are the following: (i) The particle $r_{0}$ can only exist inside a particle $q_{0}, q_{+}$, or $q_{-}$. Therefore freezing out the latter three will freeze out the former even if its energy remains finite. (ii) The particle $q_{0}$ comes in two parity-violating versions. In the context of $\mathcal{H}_{1}$ or other models where the two configurations $\uparrow \circ \downarrow$ and $\downarrow \circ \uparrow$ have the same energy, they can be treated as identical particles. However, in situations where the two configurations have to be 


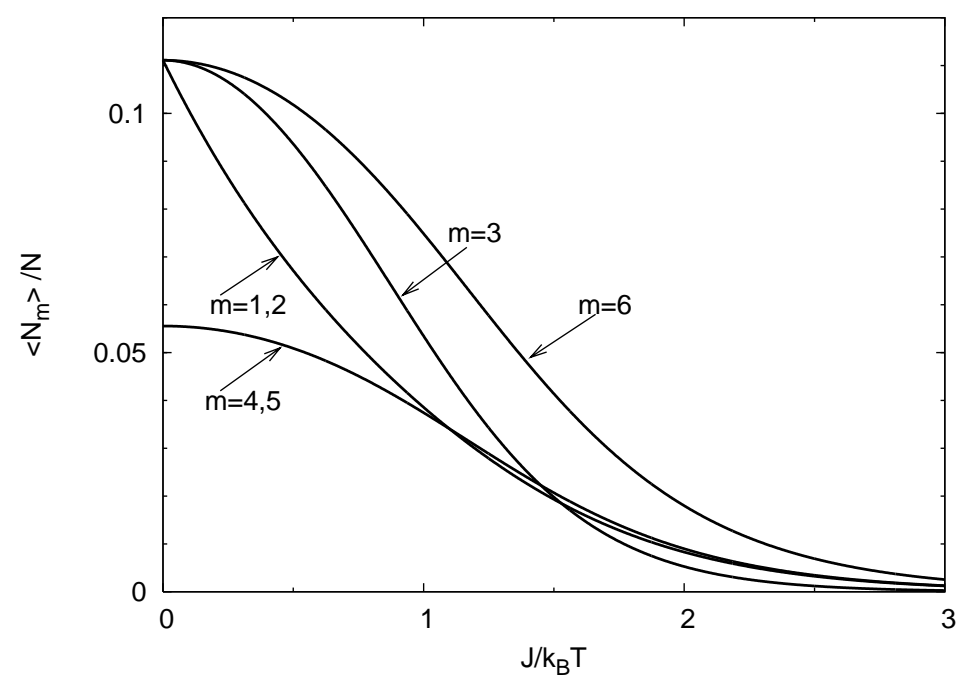

Figure 2. Average numbers $\left\langle N_{m}\right\rangle / N$ of soliton-pair particles (per site) versus inverse temperature $J / k_{B} T$ of $\mathcal{H}_{1}$.

assigned different energies we must treat them as belonging to different species and determine their statistical interaction with each other and with all the other particle species. (iii) A spin interaction beyond nearest neighbors added to $\mathcal{H}_{1}$ will, in general, produce a coupling between the particles listed in Table 5 .

\section{Domains and nested domains}

Here we consider $\mathcal{H}_{s}$ for $s=1 / 2,1, J<0$, and $h \leq 0$. In the following we describe how the entire spectrum is systematically generated from the ferromagnetic ground state $|\uparrow \uparrow \cdots \uparrow\rangle$ by domains $(s=1 / 2)$ or nested domains $(s=1)$ of flipped spins. These domains are independent particles subject to a statistical interaction. The thermodynamic analysis of domains is then carried out for the $s=1 / 2$ case at $h=0$.

\subsection{Combinatorics of domains}

In the notation used here, $\left\{\left|\sigma_{1} \cdots \sigma_{N}\right\rangle\right\}_{m}^{r}$ represents the set of $m$ product vectors with $r$ flipped spins that are generated from $\left|\sigma_{1} \cdots \sigma_{N}\right\rangle$ via translations. The $2^{N}=16$ states for $N=4$ in this representation are

$$
\begin{array}{lll}
\{\mid \uparrow \uparrow \uparrow \uparrow \uparrow\}_{1}^{0}, & \{|\uparrow \uparrow \uparrow \downarrow\rangle\}_{4}^{1}, & \{|\uparrow \uparrow \downarrow \downarrow\rangle\}_{4}^{2}, \\
\{|\uparrow \downarrow \uparrow \downarrow\rangle\}_{2}^{2}, & \{|\uparrow \downarrow \downarrow \downarrow\rangle\}_{4}^{3}, & \{|\downarrow \downarrow \downarrow \downarrow\rangle\}_{1}^{4} .
\end{array}
$$

The first among them is the (non-degenerate) ground state of $\mathcal{H}_{1 / 2}$ with $J<0$ and $h<0$. Domains are strings of $\mu$ consecutive down-spins. In (5.1) the states in the second set contain one 1-string, and the state in the fourth set two 1-strings. The states in the third, fifth, and sixth set contain one string with $\mu=2,3,4$, respectively. Each string of length $\mu$ contributes the amount $J+\mu h$ to the energy of the state. An Ising chain of length $N$ can thus accommodate strings with $\mu=1, \ldots, N-1$, which are treated here as distinct species of independent particles. The lone state containing 
one string with $\mu=N$ is exceptional in several respects, ignorable in macroscopic systems.

What is the number of product eigenstates that contain a configuration $\left\{N_{\mu}\right\}$ of strings? Since there must be at least one up-spin between successive strings, only those configurations can be realized which satisfy the constraint

$$
\sum_{\mu=1}^{N-1}(\mu+1) N_{\mu} \leq N
$$

We found that the number of states with given string configuration is determined by the multiplicity expression

$$
\begin{aligned}
& W\left(\left\{N_{\mu}\right\}\right)=\frac{N}{N-r} \prod_{\mu=1}^{N-1}\left(\begin{array}{c}
d_{\mu}+N_{\mu}-1 \\
N_{\mu}
\end{array}\right), \\
& d_{\mu}=N-\mu-\sum_{\mu^{\prime}=1}^{N-1} g_{\mu \mu^{\prime}}\left(N_{\mu^{\prime}}-\delta_{\mu \mu^{\prime}}\right),
\end{aligned}
$$

where

$$
g_{\mu \mu^{\prime}}=\left\{\begin{array}{ll}
\mu^{\prime}, & \mu<\mu^{\prime}, \\
\mu^{\prime}+1, & \mu \geq \mu^{\prime}
\end{array}, \quad r \doteq \sum_{\mu=1}^{N-1} \mu N_{\mu} .\right.
$$

\subsection{Combinatorics of nested domains}

The concept of nested quasiparticles in lattice models is well-known in the context of the Bethe ansatz as applied, for example, to the Hubbard model or to integrable spin-1 models [8, 11, 12, 13, 14, 15, 16]. The nested particles in Ising product states have a particularly simple structure.

In the context of $\mathcal{H}_{1}$ the nesting involves two shells. The particles on the outer shell ( $\mu$-strings) are structurally identical to the strings of $\mathcal{H}_{1 / 2}$. We start from the $\mu$-string vacuum, $\{|\uparrow \uparrow \cdots \uparrow\rangle\}_{1}^{0}$, and generate a total of $2^{N}$ product states composed of site variables $\uparrow$ and $\circ$. On the inner shell we take any $\mu$-string of the outer shell and use it as the vacuum for $\nu$-strings. Hence a $\nu$-string is a sequence of $\nu$ successive $\downarrow$-sites embedded in a region of $\mu$ o-sites between consecutive $\uparrow$-sites. Naturally, we

must have $\nu \leq \mu$. This prescription is illustrated in Table 7 for $N=4$. The two-shell nesting of string particles leads to the multiplicity expression

$$
\begin{aligned}
W\left(\left\{N_{\mu}\right\},\left\{N_{\nu}^{(\mu)}\right\}\right)= & \frac{N}{N-r} \prod_{\mu}\left(\begin{array}{c}
d_{\mu}+N_{\mu}-1 \\
N_{\mu}
\end{array}\right) \\
& \times \frac{\mu}{\mu-r_{\mu}} \prod_{\nu}\left(\begin{array}{c}
d_{\nu}^{(\mu)}+N_{\nu}^{(\mu)}-1 \\
N_{\nu}^{(\mu)}
\end{array}\right)
\end{aligned}
$$

with $d_{\mu}$ from (5.4), $g_{\mu \mu^{\prime}}, r$ from (5.5), and

$$
d_{\nu}^{(\mu)}=\mu-\nu-\sum_{\nu^{\prime}} g_{\nu \nu^{\prime}}\left(N_{\nu^{\prime}}^{(\mu)}-\delta_{\nu \nu^{\prime}}\right), \quad r_{\mu}=\sum_{\nu} \nu N_{\nu}^{(\mu)}
$$

As in previous applications, there are instances (ignorable for macroscopic systems) where expression (5.6) is inapplicable. 
Table 7. Nested-string interpretation of the product eigenstates for $N=4$ in $\mathcal{H}_{1}$. The $2^{N}$ states on the left represent the outer shell of the nesting. Each $\mu$-string is underlined and serves as the vacuum for $\nu$-strings on the inner shell. From each of the $2^{N}$ effective $\mathcal{H}_{1 / 2}$ product states on the left are thus generated one or several $\mathcal{H}_{1}$ product states on the right for a total of $3^{N}$.

\begin{tabular}{rl|lr}
\hline 1 & $\{|\uparrow \uparrow \uparrow \uparrow\rangle\}_{1}^{+4}$ & $\{|\uparrow \uparrow \uparrow \uparrow\rangle\}_{1 \times 1}^{+4}$, & 1 \\
4 & $\{|\uparrow \uparrow \uparrow o\rangle\}_{4}^{+3}$ & $\{|\uparrow \uparrow \uparrow \circ\rangle\}_{4 \times 1}^{+3},\{|\uparrow \uparrow \uparrow \downarrow\rangle\}_{4 \times 1}^{+2}$, & 8 \\
4 & $\{|\uparrow \uparrow \circ 0\rangle\}_{4}^{+2}$ & $\{|\uparrow \uparrow \circ 0\rangle\}_{4 \times 1}^{+2},\{|\uparrow \uparrow \downarrow \circ\rangle\}_{4 \times 2}^{+1},\{\mid \uparrow \uparrow \uparrow \downarrow \downarrow\}_{4 \times 1}^{0}$, & 16 \\
4 & $\{|\uparrow \circ \circ \circ\rangle\}_{4}^{+1}$ & $\{|\uparrow \circ \circ \circ\rangle\}_{4 \times 1}^{+1},\{|\uparrow \downarrow \circ \circ\rangle\}_{4 \times 3}^{0},\{|\uparrow \downarrow \downarrow \circ\rangle\}_{4 \times 3}^{-1},\{|\uparrow \downarrow \downarrow \downarrow\rangle\}_{4 \times 1}^{-2}$ & 32 \\
1 & $\{|\underline{\circ} \circ \circ\rangle\}_{1}^{0}$ & $\{|\circ \circ \circ \circ\rangle\}_{1 \times 1}^{0},\{|\downarrow \circ \circ \circ\rangle\}_{1 \times 4}^{-1}$ & \\
& & $\{|\downarrow \downarrow \circ \circ\rangle\}_{1 \times 4}^{-2},\{|\downarrow \circ \downarrow \circ\rangle\}_{1 \times 2}^{-2},\{|\downarrow \downarrow \downarrow \circ\rangle\}_{1 \times 4}^{-3},\{|\downarrow \downarrow \downarrow \downarrow\rangle\}_{1 \times 1}^{-4}$, & 16 \\
2 & $\{|\uparrow \circ \uparrow \circ\rangle\}_{2}^{+2}$ & $\{|\uparrow \circ \uparrow \circ\rangle\}_{2 \times 1}^{+2},\{|\uparrow \downarrow \uparrow \circ\rangle\}_{4 \times 1}^{+1},\{|\uparrow \downarrow \uparrow \downarrow\rangle\}_{2 \times 1}^{0}$, & 8 \\
\hline 16 & & & 81
\end{tabular}

\subsection{Statistical mechanics of domains}

Returning to $\mathcal{H}_{1 / 2}$ with $J<0$ and setting $h=0$, we now derive the exact thermodynamics of a system of strings via the method outlined in Sec. 2. It is evident from Wu's derivation [10] of Eqs. (2.5) that their applicability in the present context is limited to situations where the system has a large capacity for strings of all sizes that are permitted. To circumnavigate this restriction we introduce a limit on the length of allowed strings, $\mu \leq M \ll N$. The thermodynamic limit of $\mathcal{H}_{1 / 2}$ requires that we set $N \rightarrow \infty$ before setting $M \rightarrow \infty$.

With the specifications regarding statistical interaction of strings from Sec. 5.1 we write for the grand potential the expression

$$
\Omega_{M}(K)=-k_{B} T \sum_{\mu=1}^{M} A_{\mu} \ln \left(\frac{w_{\mu}+1}{w_{\mu}}\right), \quad A_{\mu}=N-\mu,
$$

where the $w_{\mu}$ satisfy

$$
4 K=\ln \left(w_{\mu}+1\right)-\sum_{\mu^{\prime}=1}^{M} g_{\mu^{\prime} \mu} \ln \frac{w_{\mu^{\prime}}+1}{w_{\mu^{\prime}}}, \quad K=\frac{|J|}{4 k_{B} T} .
$$

The transformation of variable, $\xi_{\mu} \doteq \ln \left(w_{\mu}+1\right)$, turns Eqs. (5.8) and (5.9) into

$$
\begin{aligned}
& \Omega_{M}(K)=\frac{|J|}{4 K} \sum_{\mu=1}^{M}(N-\mu) \ln \left(1-e^{-\xi_{\mu}}\right), \\
& \xi_{\mu}=4 K-\mu \sum_{\mu^{\prime}=1}^{M} \ln \left(1-e^{-\xi_{\mu^{\prime}}}\right)-\sum_{\mu^{\prime}=\mu}^{M} \ln \left(1-e^{-\xi_{\mu^{\prime}}}\right) .
\end{aligned}
$$

Introducing the quantity

$$
\Phi_{\mu} \doteq-\frac{1}{4 K} \sum_{\mu^{\prime}=1}^{\mu} \ln \left(1-e^{-\xi_{\mu^{\prime}}}\right)
$$


we rewrite (5.11) in the form

$$
\xi_{\mu}=4 K\left[1+(\mu+1) \Phi_{M}-\Phi_{\mu-1}\right] .
$$

This sets the stage for determining $\Phi_{M}$ via a recursive scheme:

$$
\begin{aligned}
& \Phi_{1}=-\frac{1}{4 K} \ln \left(1-q^{1+2 \Phi_{M}}\right), \quad q \doteq e^{-4 K}, \\
& \Phi_{2}=\Phi_{1}-\frac{1}{4 K} \ln \left(1-\exp \left(-4 K\left(1+3 \Phi_{M}\right)-\ln \left(1-q^{1+2 \Phi_{M}}\right)\right)\right),
\end{aligned}
$$

leading to

$$
\Phi_{\mu}=-\frac{1}{4 K} \ln \left(1-q^{1+2 \Phi_{M}} \frac{1-q^{\mu \Phi_{M}}}{1-q^{\Phi_{M}}}\right) .
$$

Setting $\mu=M$ we arrive at a polynomial equation for $q^{\Phi_{M}}$ :

$$
q q^{(M+2) \Phi_{M}}+(1-q) q^{2 \Phi_{M}}-2 q^{\Phi_{M}}+1=0 .
$$

The solution of (5.15) substituted into (5.10) via (5.14) and (5.13) determines the grand potential of a system of strings with maximum length $M$ in a chain of $N$ sites with $M \ll N$. Taking the limit $N \rightarrow \infty$ while keeping $M$ finite we have

$$
\omega_{M}(K) \doteq \lim _{N \rightarrow \infty} \frac{1}{N} \Omega_{M}(K)=\frac{|J|}{4 K} \sum_{\mu=1}^{M} \ln \left(1-e^{-\xi_{\mu}}\right)=-|J| \Phi_{M} .
$$

If we now take the limit $M \rightarrow \infty$, the first term in Eq. (5.15) vanishes, and the solution,

$$
q^{\Phi_{\infty}}=(1+\sqrt{q})^{-1},
$$

substituted into (5.16), yields

$$
\omega_{\infty}(K)=-\frac{|J|}{4 K} \ln \left(1+e^{-2 K}\right),
$$

which is indeed the exact result for $\mathcal{H}_{1 / 2}$ with $h=0, J<0$ and the string vacuum at the origin of the energy scale.

The statistical mechanics of a system of domains with maximum length $M$ on a lattice of $N$ sites may very well be of interest in a number of contexts outside magnetism. We have reduced the problem to solving a polynomial equation of degree $M+1$. Consider the entropy per site of strings with $\mu \leq M$ on an infinite lattice, $s_{M}(K) \doteq \lim _{N \rightarrow \infty} S_{M}(K) / N$, inferred from (5.16). Compact analytic solutions are readily calculated for $M=1$ (one-strings only) and $M=\infty$ (all strings allowed):

$$
\begin{aligned}
& \frac{s_{1}(K)}{k_{B}}=\ln \left(\frac{\sqrt{1+4 e^{-4 K}}+1}{2}\right)+\frac{8 K e^{-4 K}}{1+4 e^{-4 K}+\sqrt{1+4 e^{-4 K}}}, \\
& \frac{s_{\infty}(K)}{k_{B}}=\ln \left(1+e^{-2 K}\right)+\frac{2 K}{e^{2 K}+1} .
\end{aligned}
$$

Entropy curves for several $M$ are shown in Fig. 3. As we relax the restriction on the length of permissible domains, the entropy at any given nonzero temperature becomes larger. The relative contribution of longer domains is larger at low $T$ than at high $T$. All domains have the same energy. With $T$ increasing, the longer domains tend to be crowded out by the shorter ones. As the restriction on length is lifted altogether, the Ising result $(M=\infty)$ is approached from below.

The same type of analysis is applicable to any model with spin- $1 / 2$ Ising product eigenstates and with arbitrary energy values $\epsilon_{m}, \mu=1,2, \ldots, M$ assigned to the domains. The left-hand side of Eq. (5.9) must then be replaced by $4 K_{\mu}$, $K_{\mu}=\epsilon_{\mu} / 4 k_{B} T$. For $\mathcal{H}_{1 / 2}$ at $h \neq 0$ we must use $\epsilon_{\mu}=J+\mu h$. 


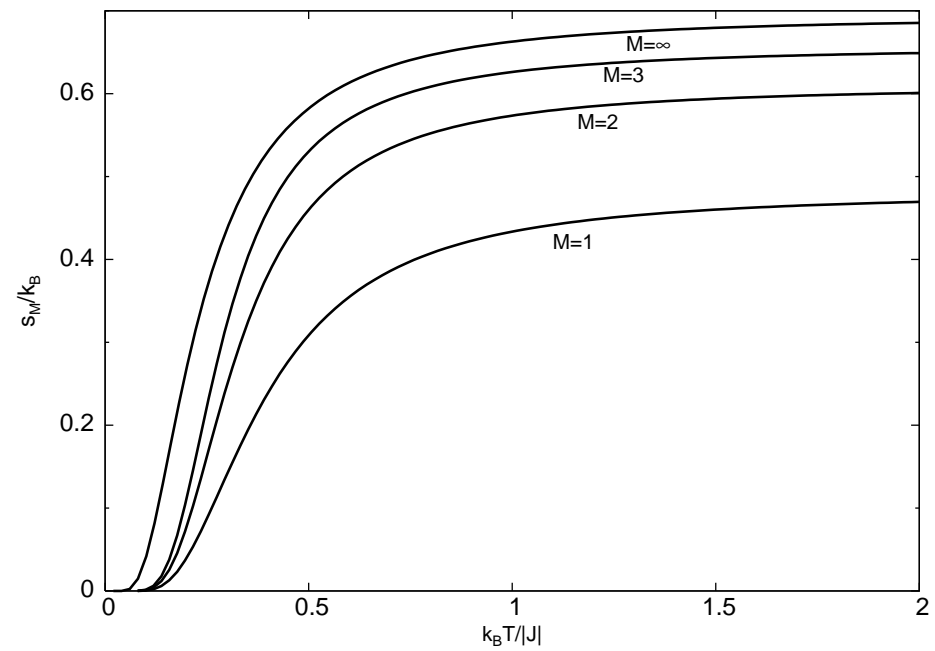

Figure 3. Entropy per site for $N \rightarrow \infty$ versus reduced temperature of a system of domains with maximum length $M$. The case $M=\infty$ represents $\mathcal{H}_{1 / 2}$.

\subsection{Distribution of domains}

What is the relative frequency of occurrence of domains of size $\mu$ for given maximum size $M$ at temperature $T$ in an infinite chain? To answer this question we adapt Wu's linear equations (2.6) to the situation at hand:

$$
w_{\mu}\left\langle n_{\mu}\right\rangle+\sum_{\mu^{\prime}=1}^{M} \mu^{\prime}\left\langle n_{\mu^{\prime}}\right\rangle+\sum_{\mu^{\prime}=1}^{\mu}\left\langle n_{\mu^{\prime}}\right\rangle=1, \quad \mu=1, \ldots, M,
$$

where $n_{\mu} \doteq N_{\mu} / N$ and where we have ignored a contribution of $\mathrm{O}(\mu / N)$ to the right-hand side by effectively taking the limit $N \rightarrow \infty$, while keeping $M$ finite. The quantities $w_{\mu}=e^{\xi_{\mu}}-1$ are known from the solution of (5.15) via (5.14) and (5.13).

Here we carry out the calculation for the case $M \rightarrow \infty$. The solution (5.17) substituted into (5.14) yields

$$
q^{\Phi_{\mu}}=\frac{1}{1+\sqrt{q}}\left(1+\frac{\sqrt{q}}{(1+\sqrt{q})^{\mu}}\right) \quad(M=\infty),
$$

which, upon substitution in (5.13), produces the $w_{\mu}$ needed in (5.21):

$$
w_{\mu}=\frac{1}{\sqrt{q}}+\frac{(1+\sqrt{q})^{\mu}}{q} \quad(M=\infty) .
$$

Now we rewrite Eqs. (5.21) in the form

$$
w_{\mu}\left\langle n_{\mu}\right\rangle+\sum_{\nu=1}^{\mu}\left\langle n_{\nu}\right\rangle=\zeta, \quad \mu=1,2, \ldots
$$

where the quantity

$$
\zeta \doteq 1-\sum_{\nu=1}^{\infty} \nu\left\langle n_{\nu}\right\rangle
$$


can be treated as a constant to be determined self-consistently at the end. The solution of Eqs. (5.24), obtained by induction, is

$$
\left\langle n_{\mu}\right\rangle=\frac{P_{\mu}}{w_{\mu}}\left(1+\sum_{\nu=1}^{\infty} \frac{\nu}{w_{\nu}} P_{\nu}\right)^{-1}, \quad P_{\mu} \doteq \prod_{\nu=1}^{\mu} \frac{w_{\nu}}{w_{\nu}+1},
$$

and, after normalization,

$$
\left\langle\hat{n}_{\mu}\right\rangle \doteq\left\langle n_{\mu}\right\rangle\left(\sum_{\nu=1}^{\infty}\left\langle n_{\nu}\right\rangle\right)^{-1}=\frac{P_{\mu}}{w_{\mu}}\left(\sum_{\nu=1}^{\infty} \frac{P_{\nu}}{w_{\nu}}\right)^{-1}=\frac{P_{\mu}}{w_{\mu}\left(1-P_{\infty}\right)},
$$

From (5.12) and (5.26) we infer that $P_{\mu}=q^{\Phi_{\mu}}$ a quantity evaluated in (5.22). The assembly of the ingredients (5.22), (5.23), and (5.17) to expression (5.27) produces the following explicit result for the distribution of lengths $\mu$ of string particles in $\mathcal{H}_{1 / 2}$ at temperature $T$ and zero magnetic field:

$$
\left\langle\hat{n}_{\mu}\right\rangle=\frac{\sqrt{q}}{(1+\sqrt{q})^{\mu}}=\frac{e^{-2 K}}{\left(1+e^{-2 K}\right)^{\mu}}, \quad \mu=1,2, \ldots
$$

This is a realization of Pascal's distribution, $P(\mu)=\gamma(1-\gamma)^{\mu-1}$, if we set $\gamma=$ $e^{-K} /\left(e^{K}+e^{-K}\right)$. This result was previously derived by Denisov and Hänggi [3] using a very different method in their study of finite Ising chains with open boundaries. This distribution indeed favors short strings in the crowded conditions at high $T$, in agreement with observations made in our discussion of the entropy curves (Fig. 3). At low $T$ the distribution is flat, consistent with the fact that all strings have the same energy. With some additional effort our solution can be generalized to finite $M$, and to models with arbitrary values for the energies $\epsilon_{\mu}$ of domains of size $\mu$.

\section{Conclusion}

We have demonstrated that the conceptual framework of statistical interaction between quasiparticles in many-body systems 9 , 10, leads to significant new insights into the statistical mechanics of Ising chains and related models with spin- $1 / 2$ or spin1 product eigenstates on a one-dimensional lattice. We have identified, in particular, the nature of complementary sets of independent particles on the basis of which the spectrum of Ising chains with $s=1 / 2$ and $s=1$ can be generated systematically from either the ferro- or antiferromagnetic ground state.

The Néel state is the vacuum for solitonic particles. In the $s=1 / 2$ case the solitons themselves are the independent particles. They are antiferromagnetic domain walls, confined to single bonds, with spin $\pm 1 / 2$ and semionic statistical interaction. In the $s=1$ case the solitons are merely building blocks of particles. All independent particles are soliton pairs. The paired solitons may be on the same bond or on bonds any number of lattice units apart. We have carried out the exact statistical mechanical analysis of solitons (two species) for $s=1 / 2$ and of soliton pairs (six species) for $s=1$.

The state with all spins up is the vacuum for string particles. In the $s=1 / 2$ case the independent particles are domains of overturned spins and in the $s=1$ case they are nested domains, i.e. domains inside domains of halfway overturned spins. By working out their exact statistical interaction we have set the stage for the statistical mechanical analysis of domains and nested domains. We have carried out that analysis for the $s=1 / 2$ case and established contact with previous work based on different methods [3]. 
The work presented here opens up numerous opportunities for extensions and comparisons including the following. (i) The methodology developed in Secs. 3 and 4 for the identification and specification of independent solitonic particles looks promising for applications to Ising chains with $s>1$ and to Ising ladders. Preliminary results for $\mathcal{H}_{3 / 2}$, for example, indicate that the independent solitonic particles contain at least two and no more than six solitons. This again includes particles confined to one bond and particles spread across many bonds with more than one species of spacer particles acting as deconfinement agents. (ii) A question of considerable interest is how the methodology developed here can be generalized to situations with Ising interactions beyond nearest neighbors, which, in general, leads to a coupling between solitonic particles and between string particles. (iii) There exist integrable spin chain models with a parametric Ising limit. Consequently, the solitonic particles analyzed here must exist in some variant form away from the Ising limit of those models. One such link (to the spinons of the $s=1 / 2 X X Z$ model) is outlined in Appendix A. Corresponding links are bound to exist between the string and nested-string particles of Ising chains and the string solutions of the Bethe ansatz applied to integrable spin models with $s=1 / 2$ [17, 18, 19, 20, 21, 22] and $s=1$ [11, 12, 13, 14, 15, 16] near their Ising limits.

\section{Appendix A. Solitons versus spinons}

The ground state of the $s=1 / 2 X X Z$ model,

$$
\mathcal{H}_{X X Z}=\sum_{n=1}^{N}\left[J_{\perp}\left(S_{n}^{x} S_{n+1}^{x}+S_{n}^{y} S_{n+1}^{y}\right)+J_{z} S_{n}^{z} S_{n+1}^{z}\right],
$$

at $J_{\perp}, J_{z} \geq 0$ for even $N$ is non-degenerate except in the Ising limit $\left(J_{\perp}=0\right)$. The finite-size gap is of $\mathrm{O}\left(N^{-1}\right)$ in the planar regime $\left(J_{\perp}>J_{z}\right)$ and of $\mathrm{O}\left(e^{-N}\right)$ in the axial regime $\left(J_{\perp}<J_{z}\right)$. The lowest energy level in both regimes has been identified as the (unique) vacuum of spinons [22. The two lowest levels, again in both regimes, can be identified as the (twofold) vacuum of solitons

Spinons and solitons have similar but not identical semionic exclusion statistics. The similarities and differences are encoded in the multiplicity expressions. Equations (3.3)-(3.4) for solitons are to be compared with

$$
\begin{aligned}
& W_{S}\left(N_{+}, N_{-}\right)=\prod_{\sigma= \pm}\left(\begin{array}{c}
d_{\sigma}+N_{\sigma}-1 \\
N_{\sigma}
\end{array}\right), \\
& d_{\sigma}=\frac{1}{2}(N+1)-\frac{1}{2} \sum_{\sigma^{\prime}}\left(N_{\sigma^{\prime}}-\delta_{\sigma \sigma^{\prime}}\right)
\end{aligned}
$$

for spinons [9]. Away from the Ising limit, solitons (and spinons) are dispersive and scatter off each other elastically. Both kinds of particles are most conveniently identified by their momentum quantum numbers. Every $X X Z$ eigenstate has a unique spinon composition and a unique soliton composition. The relation between the spinon composition and the soliton composition is most transparent in the $X X \operatorname{limit}\left(J_{z}=0\right)$.

In Ref. 23. a motif was developed that relates the configuration of (free) JordanWigner fermions with the configuration of spinons. This motif is reproduced in Fig. A1 for $N=4$ (16 eigenstates) and amended to also show the soliton configuration. The

$\ddagger$ The names attributed to quasiparticles in quantum spin chains vary among authors. Our usage is common but not universal. 
allowed fermion momenta (in units of $\pi / N$ ) are

$$
\bar{m}_{i} \in \begin{cases}\{1,3, \ldots, 2 N-1\} & \text { for even } N_{F} \\ \{0,2, \ldots, 2 N-2\} & \text { for odd } N_{F}\end{cases}
$$

and the allowed spinon orbital momenta (in units of $\pi / N$ ) are

$$
m_{i}=\frac{N_{S}}{2}, \frac{N_{S}}{2}+2, \ldots, N-\frac{N_{S}}{2},
$$

where $N_{F}$ is the number of fermions and $N_{S}=N_{+}+N_{-}$the number of spinons in any given $X X$ eigenstate.

The exact spinon configuration is encoded in the fermion configuration as described in the following: (i) Consider the the gray fork as dividing the fermion momentum space into two domains, the inside and the outside. The outside domain wraps around at the extremes $\left(\bar{m}_{i}=N \bmod N=0\right)$. (ii) Every fermionic hole (open circle) inside represents a spin-up spinon (square surrounding open circle) and

\begin{tabular}{|c|c|c|c|c|c|c|}
\hline \multicolumn{7}{|c|}{$\overline{\mathrm{m}}_{\mathrm{i}}$} \\
\hline E & $\mathrm{k}$ & & $\begin{array}{ll}2 & 4 \\
\perp & \perp \\
\end{array}$ & $\begin{array}{l}6 \\
\perp \perp \perp \\
\end{array}$ & spinons & solitons \\
\hline 0.000 & 0 & & 0 & 이잉 & $2+, 2+, 2+, 2+$ & $2+, 2+, 2+, 2+$ \\
\hline 0.000 & 1 & & - 0 & $0:$ & $1+, 1+$ & $1+, 1+$ \\
\hline-1.000 & 2 & & ○ • & 0 ; & $1+, 3+$ & $1+, 3+$ \\
\hline 0.000 & 3 & & 00 & $\bullet$ & $3+, 3+$ & $3+, 3+$ \\
\hline 1.000 & 0 & $\bullet$ & ○ & $0 ;$ & $2+, 2+, 2+, 2-$ & $2+, 2+$ \\
\hline-1.414 & 0 & c & $\bullet$ & - 0 & --- & --- \\
\hline 0.000 & 1 & c & $\bullet$ & 0 & $1+, 1-$ & $1+, 1-$ \\
\hline 0.000 & 2 & 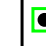 & $\bullet$ & \begin{tabular}{l|l}
0 & 0
\end{tabular} & $1+, 3-$ & --- \\
\hline 0.000 & 2 & c & 0 & - $\bullet$ & $3+, 1-$ & $3+, 1-$ \\
\hline 0.000 & 3 & 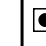 & 0 & - 0 & $3+, 3-$ & $3+, 3-$ \\
\hline 1.414 & 0 & C & 0 & 0 & $2+, 2+, 2-, 2-$ & $2+, 2-$ \\
\hline 0.000 & 1 & $\bullet$ & $\circ \cdot \bullet$ & $\bullet$ & $1-, 1-$ & $1-, 1-$ \\
\hline-1.000 & 2 & $\circ$ & $\bullet \bullet$ & $\bullet$ & $1-, 3-$ & $1-, 3-$ \\
\hline 0.000 & 3 & $\bullet$ & $\bullet \bullet$ & 0 & $3-, 3-$ & $3-, 3-$ \\
\hline 1.000 & 0 & 0 & $\bullet$ & $\square$ & $2+, 2-, 2-, 2-$ & $2-, 2-$ \\
\hline 0.000 & 0 & & 0 & $\bullet \bullet$ & $2-, 2-, 2-, 2-$ & $2-, 2-, 2-, 2-$ \\
\hline
\end{tabular}
every fermionic particle (full circle) outside represents a spin-down spinon (square

Figure A1. Fermion configurations of all eigenstates for $N=4$ of the $X X$ model $\left(J_{z}=0\right)$. Fermionic particles (holes) are denoted by full (open) circles. Spinons with spin up (down) are denoted by (black or gray) squares around open (full) circles. Solitons with spin up (down) are denoted by black squares around open (full) circles. The fermion momenta $\bar{m}_{i}$ (in units of $\pi / N$ ) can be read off the diagram. The spinon orbital momenta $m_{i}$ (also in units of $\pi / N$ ) and the spinon spins $\sigma_{i}$ are given explicitly and can be inferred from the fermion configuration as explained in the text. Also given are the wave number $k$ (in units of $2 \pi / N$ ) and the energy $E$ (in units of $J_{\perp}$ ) of each eigenstate. 
surrounding full circle). (iii) Any number of adjacent spinons in the representation of Fig. A1 are in the same orbital. Two spin-up (spin-down) spinons that are separated by $\ell$ consecutive fermionic particles (holes) have quantum numbers separated by $2 \ell$. (iv) The spinon orbital momenta are sorted in increasing order from the right-hand prong of the gray fork toward the left across the inside domain and toward the right with wrap-around through the outside domain.

For the determination of the soliton content of any $X X$ eigenstate we must also consider the black fork in Fig. A1, which is shifted two units to the left. If there is any spin-up/spin-down spinon pair as identified by the rules pertaining to the gray fork that does not also satisfy the same rules with respect to the black fork, then it is omitted from the list of solitons. All such spinons are identified by gray squares in Fig. A1. The spinon vacuum is just one of two soliton vacua, the other soliton vacuum being a two-spinon state. The two soliton vacua have wave numbers differing by $\pi$. In the Ising limit of the $X X Z$ model they correspond to the symmetric and antisymmetric combinations of the two product Néel states.

\section{Acknowledgments}

Financial support from the DFG Schwerpunkt Kollektive Quantenzustände in elektronischen $1 D$ Übergangsmetallverbindungen (for M.K.) is gratefully acknowledged.

\section{References}

[1] Ising E, 1925 Z. Phys. 25253

[2] Antal T, Droz M and Rácz Z, 2004 J. Phys. A: Math. Gen.

[3] Denisov S I and Hänggi P, 2005 Phys. Rev. E 71046137

[4] Kramers H A and Wannier G H, 1941 Phys. Rev. 60252

[5] Suzuki M, Tsujiyama B and Katsura S, 1967 J. Math. Phys. 8124

[6] Korepin V E, Bogoliubov N M and Izergin A G, Quantum Inverse Scattering Method and Correlation Functions (Cambridge University Press, Cambridge, 1993)

[7] Sutherland B, Beautiful models: 70 years of exactly solved quantum many-body problems (World Scientific, Singapore, 2004)

[8] Essler F H L, Frahm H, Göhmann F, Klümper A et al., The one-dimensional Hubbard model (Cambridge University Press, 2005)

[9] Haldane F D M, 1991 Phys. Rev. Lett. 67937

[10] Wu Y S, 1994 Phys. Rev. Lett. 73922

[11] Sutherland B, 1975 Phys. Rev. B 123795

[12] Takhtajan L A, 1982 Phys. Lett. A 87479

[13] Babujian H M, 1982 Phys. Lett. A 90479

[14] Haldane F D M, 1982 J. Phys. C: Solid St. Phys. 15 L1309

[15] Sogo K, 1984 Phys. Lett. A104 51

[16] Frahm H and Stahlsmeier M, 1998 Phys. Lett. A 250293

[17] Gaudin M, 1971 Phys. Rev. Lett. 261301

[18] Takahashi M, 1991 Phys. Rev. B 4412382

[19] Johnson J D and McCoy B M, 1972 Phys. Rev. A 61613

[20] Johnson J D, 1974 Phys. Rev. A 91743

[21] Johnson J D and Bonner J C, 1980 Phys. Rev. B 22251

[22] Takahashi M, Thermodynamics of one-dimensional Solvable Models (Cambridge University Press, Cambridge, United Kingdom, 1999)

[23] Arikawa M, Karbach M, Müller G and Wiele K, 2006 J. Phys. A: Math. Gen. 3910623 